\title{
Discourse/Conversation Analysis and Autism Spectrum Disorder
}

\author{
Michelle O'Reilly ${ }^{1} \cdot$ Jessica Nina Lester $^{2} \cdot$ Tom Muskett $^{3}$
}

Published online: 28 November 2015

(c) Springer Science+Business Media New York 2015

Qualitative research focused on autism spectrum disorders (ASD) is important, as it can refine understandings of particular issues related to ASD and be practically relevant provided that quality criteria are applied (Bölte 2014). Employing qualitative methods for the study of ASD is an important way of giving a 'voice' to participants and can be particularly useful for empowering vulnerable or disadvantaged people groups (Peters 2010). The field of mental health and disability generally is one that is mediated through language (Brown et al. 1996) and qualitative methods tend to focus on language use, specifically those using discourse or conversation analysis approaches. Quantitative evidence, whatever its form, provides important and valuable evidence about ASD. However, it is crucial that the field does not solely rely on a relatively narrow range of methodologies and forms of knowledge to inform us about such a complex and diverse condition. Qualitative methods are able to go beyond establishing the likelihood of associations between variables, towards

Michelle O'Reilly

mjo14@leicester.ac.uk; mjo14@le.ac.uk

Jessica Nina Lester

jnlester@indiana.edu

Tom Muskett

T.A.Muskett@leedsbeckett.ac.uk

1 College of Medicine, Biological Sciences, and Psychology, The Greenwood Institute, University of Leicester, Westcotes Drive, Leicester LE3 0QU, UK

2 School of Education, Inquiry Methodology, Indiana University, W.W. Wright Education Building, Bloomington, IN 47405-1006, USA

3 School of Social, Psychological and Communication Sciences, Leeds Beckett University, Calverley Building, City Campus, Leeds LS1 3HE, UK understanding the nature of such associations and the complex processes that they may be interpreted to represent. More specifically, qualitative methods are able to go beyond what works to show how and why a particular practice is effective (Rhodes 2011), particularly when taking into account established quality criteria.

In this special section, we focus specifically on discourse analysis and conversation analysis, which are languageoriented qualitative approaches that analyse what people actually do and say, as opposed to what they report that they do or say (McCabe 2006). The five included articles use discourse or conversation analysis in varying ways, and highlight how these analytic methods pursue robust validation procedures and focus on identifying systematic patterns of interaction grounded in natural data (McCabe et al. 2002) with built in quality criteria. Typically researchers applying discourse or conversation analysis use extensive corpora of audio- or video-recordings of social interactions collected in natural settings (e.g., clinic, therapy session). Analysis then involves a close, inductive examination of the interactions of all the participants in that setting. Although discourse analysis and conversation analysis are similar approaches, and both fall broadly within the landscape of qualitative research in that they treat language as their central focus (Bryman 2008), there are some important practical and epistemological differences which dictate the nature of the questions asked and the specific analytic methods used.

Conversation analysis (CA) is a qualitative approach that focuses on the study of interaction. The core aim of this approach is to examine the social organisation of activities that are produced in interaction through talk (Hutchby and Wooffitt 2008). In other words, CA aims to interrogate how people use language to perform certain social actions, such as excusing, inviting, complaining, 
justifying, questioning, and so on. CA was developed by Harvey Sacks in the 1960s alongside Emmanuel Schegloff and Gail Jefferson (Sacks 1992), and explores how participants negotiate meaning between themselves on a turnby-turn basis (Maynard and Clayman 2003; McCabe 2006). Those practicing CA prefer to collect data that is naturally-occurring (i.e., interactions that occur in the 'realworld' and are not specifically generated for research purposes through interviews or focus groups) (Potter 2004). CA focuses on the practices of social action and makes claims about the communication rules of participants. Thus, CA is able to identify the practices of social action, as well as the regularities of or deviances from those practices (Robinson 2007).

Discourse analysis (DA) is also a qualitative approach broadly characterised as focusing on talk and text in practice (Potter 2004). Unlike CA, which is a single language-based approach, DA has different variants and therefore it is difficult to provide a single definition of it. There are some shared features of the different types of DA, which include a focus on language, a focus on the broad ways in which accounts are constructed, and an acknowledgement of the variable ways people account for things. However, there are some broad differences, as some types of DA focus primarily on the content of the language used or issues discussed in any single interaction, whereas others attend to the structure of language and how that functions to create particular perspectives or understandings (see Lester and O'Reilly 2015; Wooffitt 1992 for an overview). Notably, the different approaches to DA are underpinned by different theoretical assumptions and thus serve to answer different types of research questions.

It is important to recognise that qualitative research, particularly DA and CA, has a great deal to offer in understanding the experiences and practices of individuals with ASD, families and practitioners; and in gaining knowledge in the areas of communication, behaviour, and social interaction. As an approach, CA is well-placed for exploring the communicative practices of those with ASD, the families communicating with that individual, professional discourse with those diagnosed, and the language employed in society when talking about ASD. In addition, the analytic focus afforded by CA enables for the identification of novel and unpredictable aspects of social interaction involving people with the diagnosis. For example, the detailed nature of conversation analytic findings enables for a micro-comparison of interactions involving typically developing individuals alone to interactions involving one or more individuals diagnosed with ASD, with differences across a range of interactional settings identified as a valuable area for future research (Hobson et al. 2012). Like CA, DA is also well-placed to facilitate the research field associated with ASD. Its focus on language and communication means that it is a useful way of examining the interactions of those diagnosed with the condition, and the more critical approaches are able to explore the disabling discourses of ASD, the powerful structures in society that operate, and the scientific rhetoric that filters through the research rubric.

Much research is undertaken in relation to language and communication in ASD. While this research is varied in terms of its focus, there are key similarities between most studies in this area; most work is focused on examining language and communication in sole relation to individuals diagnosed with ASD themselves. For example, some work attempts to categorise or quantify types of language and communication profiles and/or phenomena that may be presented by people diagnosed with ASD (e.g., Kwok et al. 2015). A greater body of work uses experimental paradigms to attempt to identify language-oriented cognitive, neurophysiological or genetic differences in people diagnosed with ASD, and then examine potential associations between these and language performance by such individuals (e.g., Boucher et al. 2008; Bartlett et al. 2014).

This kind of individual-focused work generates hypothesis-driven theory, which in turn can be suggestive of impairment-level intervention methods. In doing so, however, this type of research necessarily draws on the view of language and communication as being bound within individual, psychological competencies. This is certainly a valid perspective that underpins much work across psychology, linguistics, and developmental psychopathology, and is one that lends itself to experimental and quantitative methodologies. However, there are other aspects of, or perspectives on, language and communication that are potentially relevant for studies of ASD, and therefore may augment existing knowledge in the area. For instance, while there is much research in relation to commonalities between individuals with ASD in terms of individual aspects of language and communication when examined more or less in isolation, much less is known about what happens during social interactions involving individuals with ASD, which-by their nature-involves more than just one individual. Indeed, research from CA in particular suggests that social interaction is a richly coordinated and collaborative activity, both generated by and reliant upon multiple speakers working together (Sacks 1992).

Moving a level above this individual-focus work, little is also known about how interactions play out in the specific kinds of social contexts that people with ASD participate within, and that are relevant to developing a broader understanding of issues experienced by those with the diagnosis. For instance, it is well established that everyday social encounters differ in their organization and tacit expectations to those in classrooms, clinical interactions, 
diagnostic sessions, and so forth. What happens, therefore, when people with ASD are participants in these contexts? Moreover, how is language used by people (not just those with ASD) to talk about ASD as a concept itself? While this latter topic may feel somewhat distant from what may typically be thought of as research about 'language and ASD', identifying how ASD is talked about across different environments is evidently important for understanding broader social aspects of the diagnosis. This, therefore, opens up a further level at which to consider language, communication, and ASD - that being how the diagnosis is described and represented at a broader socio-cultural level, including within media and popular culture.

These broad levels at which language and communication in relation to ASD can be considered are diagrammatized below. theorising the condition (Sterponi et al. 2014). For example, in a recent quantitative study, Hobson et al. (2012) explored a suggested index of impaired intersubjectivity in dyadic interactions involving children with ASD by coding the extent to which the linguistic construction of turns by children with and without ASD were lexically congruent with the construction of the preceding utterance from the other speaker. Such a research focus on both the participants in interactions (as opposed to the child with ASD alone) and the sequential influence of one speaker's behaviour on another is very much consistent with the DA and CA paradigm. However, where Hobson et al.'s study sought to measure one facet of the organisation of interaction, through its qualitative underpinnings, both DA and CA enable for an entirely open and data-driven consideration of a vast range of sequential phenomena; this, in turn,

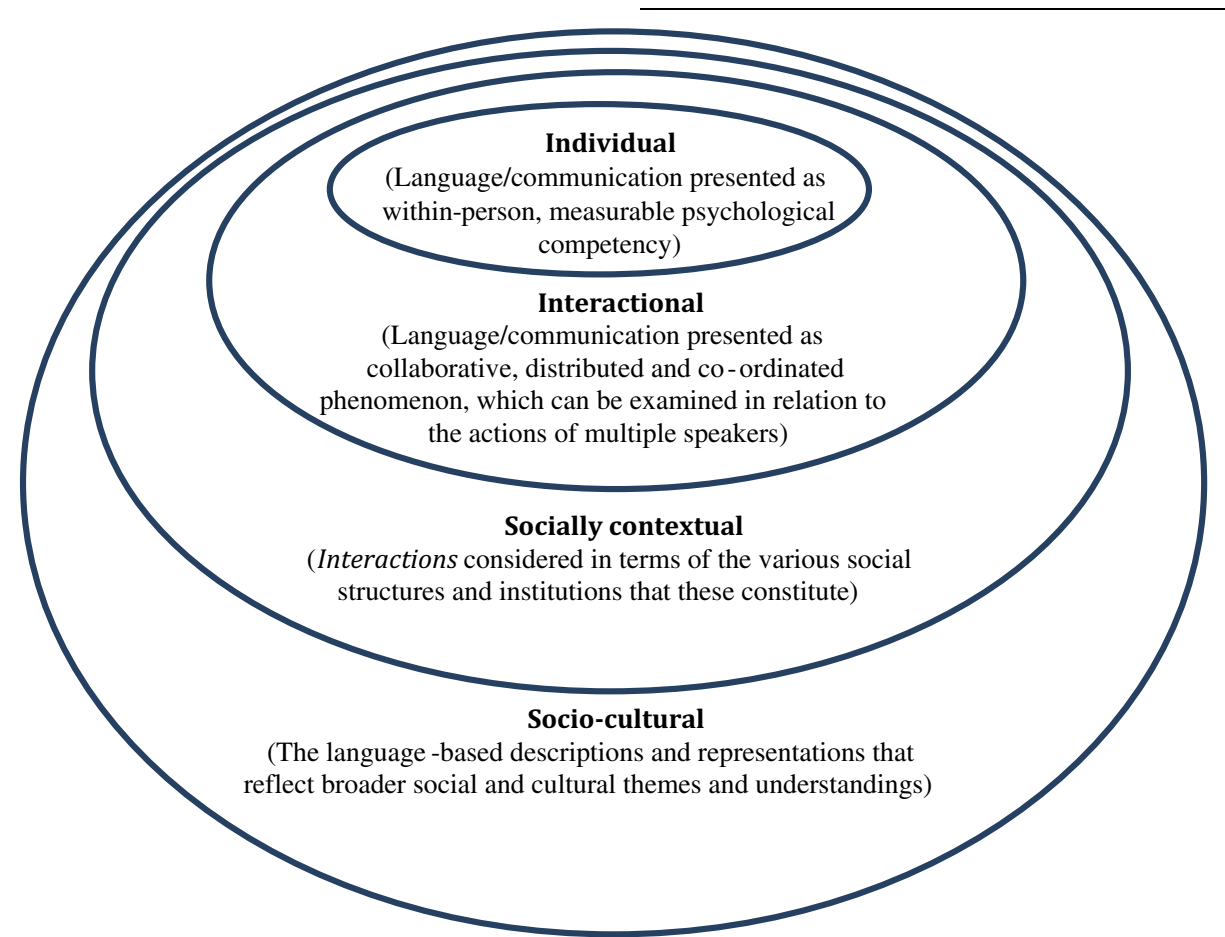

At present, there is a large repertoire of empirical work using DA or CA, illustrating how an interaction can be explained by a set of variable, yet predictable and orderly conversational principles. DA and CA are able to offer novel ideographic insights on a range of issues related to social interaction, communication, and language use in ASD that are of concern to diagnosed individuals, families and practitioners. Furthermore, close analytical attention to the interactions of those with ASD has potential to highlight areas not necessarily identified or considered in larger scale studies but directly relevant, both empirically and theoretically, for a number of contemporary approaches to provides new opportunities for the empirical examination of the theoretical basis of work, such as that by Hobson and colleagues (Sterponi et al. 2014).

Further, findings from CA studies often highlight important areas for developing randomised controlled trials in healthcare settings. For example, in relation to primary care settings, Heritage et al. (2007) noted that particular types of questions posed by doctors lead to a closing down of healthcare topics and often resulted in dissatisfaction and unmet concerns amongst patients. These types of findings open up the possibility for a large-scale trial where two different questioning types can be utilised in primary 
care settings to explore the impact on practice. This is particularly useful, as people diagnosed with ASD and their families make relevant their own understandings of the condition through their language use (Lester 2012).

In considering the applications of DA and CA findings to the field of ASD, some researchers present DA and CA findings without necessarily considering the relation to current knowledge or theories about ASD. Conversely, other researchers interpret their findings in relation to the ASD literature. In some cases, DA and CA findings can augment or triangulate existing accepted knowledge about the diagnosis (Dobbinson 2015). Some researchers have also demonstrated that DA and CA analyses can raise important questions about how conditions, such as ASD, are typically represented, particularly in terms of how predictions about language and communication arising from individually-oriented research may not play out when examined at the level of interaction or above (Sterponi et al. 2014).

With these potential contributions in mind, this special section includes five articles wherein the contributing authors illustrate how issues pertinent to children with ASD and their families can be made sense of using a DA or CA approach. In addition, each paper reports on research that utilises these analytic methods to illuminate how these approaches can and do augment existing knowledge of ASD in a way that has practical and theoretical value for the field. More specifically, across the papers the contributing authors focus on differing contexts. For instance, Bottema-Beutel et al. focus their analysis on social group interactions. Distinct from this, Solomon et al. attend to doctor patient communication and the ways in which the presentation of a problem unfolds within such an environment. Further, some of the authors, such as Maynard et al., focus on the talk of an individual child, while others, such as $\mathrm{Yu}$, attend to multi-party talk. While each of the studies is broadly situated at the intersection of the field of ASD and language and communication, each paper also offers unique insights related to key substantive issues related to children with ASD. Bottema-Beutal et al. point to insights related to social competency and morality; Solomon et al. highlight children's experiences of illness and the ways in which problems are presented; Sterponi et al. offer insights related to pronoun atypicality and echolia; Maynard et al. hone in on parental styles of interaction; and $\mathrm{Yu}$ points to the management of bilingualism within a family context. Yet, common to each of the papers is an overarching focus on the interactional level of language and communication, with Yu's piece also offering some insights specific to the socially-contextual level of language (e.g., language policy environment). Additionally, while all of the papers draw upon some type of DA or CA, there is uniqueness to the way in which this is carried out.
For example, Olga et al. offer a line by line, detailed analysis of the talk, exemplifying the very principles of $\mathrm{CA}$. Yu, on the other hand, presents a DA that also draws upon the tradition of ethnography, thereby providing a richer description of the context.

Across the papers, perhaps one of the most promising applications of the methodologies utilised in this special section is in relation to clinical and practical work. Indeed, a growing body of research, much of which has been produced by practitioners, indicates that the fastidious and socially-oriented findings generated by DA and CA approaches can underpin novel approaches for assessment, intervention, identifying therapeutic progress, reflective practice, and training. Such work has much potential relevancy for working with individuals with ASD. There are two particular approaches that we draw attention to here, which we suggest are keenly related to the five included papers.

In terms of direct practice, an area where there has been relatively advanced development of practically applied CA is with adults who have acquired language and communication difficulties following brain injury. Here, discursive approaches have enabled the generation of tools for assessment and intervention that target communication at the level of (at least) the dyad (see Beckley et al. 2013). Hence, rather than focusing on the individual with aphasia, this interactional focus has enabled practitioners to work with service users and their friends/family to improve mutual experiences of conversation and understanding of the meaning of certain communication behaviours. Evidently, such a systemic approach could be powerful when working with individuals with ASD, including in circumstances where there are frequent misunderstandings and/or manifestations of behaviour that is challenging. In terms of the latter, a CA approach in particular could enable a nuanced modelling of precursors or triggers to crisis situations, and an empirically-grounded understanding of how these could be avoided. As just one example, Sterponi et al.'s paper offers a useful set of findings that could conceivably be taken upon and made sense of in relation to assessment and intervention.

More indirectly, the analytic approaches used in this special section have been shown to possess powerful applications in the context of training. For instance, one can see how findings generated in Olga et al.'s paper might shape the ways in which medical practitioners are trained and come to make sense of their interactions with children with ASD and their families. Indeed, there have been a number of recently developed approaches, again primarily using CA, that have sought to present detailed analyses of professional-client interaction back to professionals in order to draw attention to previously tacit interactional behaviours that may be facilitating or counterproductive 
for the practice at hand (see Heritage et al. 2007; Stokoe 2014). As with possible applications of DA and CA for assessment and intervention, there are again clear opportunities for developing systemic practice around complex behaviours afforded by use of these approaches in the context of ASD. It is here that we invite the readers of the Journal of Autism and Developmental Disorders to both engage critically with the articles included within this special section and begin to envision how these types of methodologically rich work might inform the field of ASD in the future. Further, we call for ongoing dialogue around how these approaches to research may shape practice.

Author Contributions MJO developed the idea and proposed the special issue. All three editors made an equal contribution in developing and writing the editorial. TM developed the figure for use in the editorial. All three editors read and approved the final version of the manuscript.

\section{Compliance with Ethical Standards}

Conflict of interest All authors declare no conflict of interest and as this is an editorial not a research article there is no funding to declare.

Human and Animal Rights This article does not contain any studies with human participants or animals performed by any of the authors.

\section{References}

Bartlett, C. W., Hou, L., Flax, J. F., Hare, A., et al. (2014). A genome scan for loci shared by autism spectrum disorder and language impairment. American Journal of Psychiatry, 171(1), 72-81.

Beckley, F., Best, W., Johnson, F., Edwards, S., Maxim, J., \& Beeke, S. (2013). Conversation therapy for agrammatism: Exploring the therapeutic process of engagement and learning by a person with aphasia. International Journal of Language and Communication Disorders, 48(2), 220-239.

Boucher, J., Bigham, S., Mayes, A. R., \& Muskett, T. (2008). Recognition and language in low functioning autism. Journal of Autism and Developmental Disorders, 38(7), 1259-1269.

Brown, B., Nolan, P., Crawford, P., \& Lewis, A. (1996). Interaction, language and the 'narrative turn' in psychotherapy and psychiatry. Social Science and Medicine, 43(11), 1569-1578.

Bryman, A. (2008). Social research methods (3rd ed.). Oxford: Oxford University Press.

Bölte, S. (2014). The power of words: Is qualitative research as important as quantitative research in the study of autism? Autism, 18(2), 67-68.

Dobbinson, S. (2015). Conversation with an adult with features of autism spectrum disorder in secure forensic care. In M. O'Reilly
\& J. N. Lester (Eds.), The Palgrave handbook of adult mental health: Discourse and conversation studies. London: Palgrave.

Heritage, J., Robinson, J., Elliott, M., Beckett, M., \& Wilkes, M. (2007). Reducing patients' unmet concerns in primary care: the difference one word can make. Journal of General Internal Medicine, 22(10), 1429-1433.

Hobson, R. P., Hobson, J., Garcia-Pèrez, R., \& Du Bois, J. (2012). Dialogic linkage and resonance in autism. Journal of Autism and Developmental Disorders, 42, 2718-2728.

Hutchby, I., \& Wooffitt, R. (2008). Conversation analysis (2nd ed.). Cambridge: Polity Press.

Kwok, E., Brown, H., Smyth, R., \& Cardy, J. O. (2015). Metaanalysis of receptive and expressive language skills in autism spectrum disorder. Research in Autism Spectrum Disorders, 9, $202-222$.

Lester, J. (2012). A discourse analysis of parents' talk around their children's autism labels. Disability Studies Quarterly, 32(4), art. 1 .

Lester, J., \& O'Reilly, M. (2015). Is evidence-based practice a threat to the progress of the qualitative community? Arguments from the bottom of the pyramid. [Special issue; 20th anniversary edition]. Qualitative Inquiry, 21(7), 628-632.

Maynard, D., \& Clayman, S. (2003). Ethnomethodology and conversation analysis. In L. Reynolds \& N. Herman-Kinney (Eds.), Handbook of symbolic interactionism (pp. 173-202). Lanham: Altamira press.

McCabe, R. (2006). Conversation analysis. In M. Slade \& S. Priebe (Eds.), Choosing methods in mental health research: Mental health research from theory to practice (pp. 24-46). Hove: Routledge.

McCabe, R., Heath, C., Burns, T., \& Priebe, S. (2002). Engagement of patients with psychosis in the consultation: conversation analytic study. British Medical Journal, 325, 1148-1151.

Peters, S. (2010). Qualitative research methods in mental health. Evidence Based Mental Health, 13(2), 35-40.

Potter, J. (2004). Discourse analysis as a way of analysing naturally occurring talk. In D. Silverman (Ed.), Qualitative research: Theory, method and practice (2nd ed., pp. 200-221). London: Sage.

Rhodes, P. (2011). Why clinical psychology needs process research: An examination of four methodologies. Clinical Child Psychology, 17(4), 495-504.

Robinson, J. (2007). The role of numbers and statistics within conversation analysis. Communication Methods and Measures, 1(1), 65-75.

Sacks, H. (1992). Edited by Jefferson, G. Lectures in conversation. Oxford: Blackwell.

Sterponi, L., de Kirby, K., \& Shankey, J. (2014). Rethinking language in autism. Autism, doi:10.1177/1362361314537125.

Stokoe, E. (2014). The conversation analytic role-play method (CARM): A method for training communication skills as an alternative to simulated role-play. Research on Language and Social Interaction, 47(3), 255-265.

Wooffitt, R. C. (1992). Telling tales of the unexpected: The organization of factual discourse. London: Harvester/ Wheatsheaf. 